Research Article

\title{
Determination of Caffeine Content in Commercial Energy Beverages Available in Saudi Arabian Market by Gas Chromatography-Mass Spectrometric Analysis
}

\author{
Mohammed Al-Bratty, ${ }^{1}$ Hassan A. Alhazmi ${ }^{10},{ }^{1,2}$ Zia ur Rehman, ${ }^{1}$ Sadique A. Javed, ${ }^{1}$ \\ Waquar Ahsan, ${ }^{1}$ Asim Najmi, ${ }^{1}$ Gulrana Khuwaja, ${ }^{1}$ Hafiz A. Makeen, ${ }^{3}$ and Asaad Khalid ${ }^{2}$ \\ ${ }^{1}$ Department of Pharmaceutical Chemistry, College of Pharmacy, Jazan University, P.O. Box 114, Jazan 45142, Saudi Arabia \\ ${ }^{2}$ Substance Abuse and Toxicology Research Centre, Jazan University, P.O. Box 114, Jazan 45142, Saudi Arabia \\ ${ }^{3}$ Department of Clinical Pharmacy, College of Pharmacy, Jazan University, P.O. Box 114, Jazan 45142, Saudi Arabia
}

Correspondence should be addressed to Hassan A. Alhazmi; hasalhazmi@gmail.com

Received 2 March 2020; Revised 27 April 2020; Accepted 1 July 2020; Published 22 July 2020

Academic Editor: Pedro D. Vaz

Copyright (C) 2020 Mohammed Al-Bratty et al. This is an open access article distributed under the Creative Commons Attribution License, which permits unrestricted use, distribution, and reproduction in any medium, provided the original work is properly cited.

\begin{abstract}
The popularity of energy beverages among young adult population is high. These drinks are claimed to boost energy and performance and contain high concentration of caffeine as one of the several ingredients. Discrepancies have been encountered by some of the previous studies between the actual quantity of caffeine present in the product and the amount mentioned on the label, making the determination of caffeine content in these drinks very important. Thus, in this study, we analyzed the caffeine concentration in most popular energy drinks available in Saudi Arabia. The energy drink samples $(n=9)$ were procured from retail outlets. Sample solutions were prepared in methanol and analyzed for caffeine content by GC-MS. Chromatographic parameters were optimized to achieve optimum resolution and various validation parameters were evaluated. The method was successfully applied for the quantification of caffeine in energy drinks by directly injecting the multifold diluted samples in methanol. The method was linear $\left(r^{2}=0.999\right)$ over a concentration range of $5-25 \mu \mathrm{g} / \mathrm{mL}$, specific, precise (\%RSD of peak area $\left.=0.56-0.78\right)$, and accurate (\%recovery $=99.3-101.2 \%$ ). The amounts of caffeine determined were found in the range of $20.82-33.72 \mathrm{mg} / 100 \mathrm{~mL}$ $(52.05-84.3 \mathrm{mg} / \mathrm{pack})$. Results revealed that the amount of caffeine actually present in the tested drinks varied within $\pm 10 \%$ range from the amount specified on the product labels. The amounts of caffeine detected in tested beverages were within the USFDA safe upper limit of per-day caffeine consumption, which has indicated that the consumption of one serving of energy drink is unlikely to produce any adverse health effect.
\end{abstract}

\section{Introduction}

Subsequent to the introduction of Red Bull in the US market in 1997, the popularity of energy drinks has rapidly grown. Energy drinks are nonalcoholic beverages, claimed to boost energy and enhance performance. Youths and young adults aged between 18 and 34 years are the typically targeted consumers for these beverages. Energy drinks contain high contents of caffeine and sugar, in addition to other ingredients such as taurine, Ginkgo biloba leaf extract, ginseng, guarana, vitamins, amino acids, and herbal ingredients [1-3]. These products are marketed as being beneficial to health; however, there is significant concern among the health professionals regarding the risks and adverse health effects associated with these beverages. Consequently, there are plenty of discussions about the health benefits versus safety of the ingredients present in these products [4]. Clauson et al. (2008) have reported that the levels of taurine, guarana, and ginseng present in energy drinks are well below the amount needed to produce therapeutically beneficial or 
harmful effects; however, the quantities of caffeine and sugar were found to be at such a concentration that can cause adverse health effects in the consumer population [5].

The most common adverse effects associated with regular consumption of energy drinks are related to central nervous system (CNS) including insomnia, restlessness, agitation, and tremors; cardiovascular system such as tachycardia and heart palpitation; and gastrointestinal disturbances [6-8]. Investigations on young adults and adolescents have reported an association between energy drinks and some behavioral risks such as smoking, violence, and drug and alcohol usage [9-11]. Some studies have suggested that the consumption of caffeinated energy drinks along with alcohol may increase negative side effects and other risky behaviors [4]. In adults, new-onset seizures associated with high intake of energy drinks were reported by Iyadurai and Chung (2007) [12], whereas another study has reported hospitalization of individuals with history of mental illness, which might be due the consumption of energy drinks [13].

The primary concern about the health risks associated with the intake of caffeinated energy drinks is the excessive consumption of caffeine. Caffeine, a stimulant alkaloid, occurs naturally in various plants such as coffee beans, kola nuts, tea leaves, cocoa beans, and many other plants. It is one of the favorite legal drugs, used as an ingredient in beverages such as soft drinks and energy drinks as well as in pharmaceutical formulations. Due to stimulant effects on the CNS, caffeine consumption can produce headache, sleep disturbance, restlessness, tachycardia, and irritability in human beings. An individual's health can be markedly affected by sleep disturbance; hence, excessive consumption of energy drink may be partially responsible for the poor health. Intake of caffeine in excessive amount can induce psychological and physiological dependence [4, 14]. Some reports also state that the excessive intake of caffeine is associated with several other diseases such as myocardial infarction, anxiety, coronary heart disease, fibrocystic breast disease, and variety of cancers including kidney, pancreas, and urinary tract cancers $[15,16]$.

The caffeine content in soft drinks varies among the brands, which is regularly monitored by the United State Food and Drug Administration (US FDA), allowing a maximum limit of $200 \mathrm{mg} / \mathrm{L}$ [17]. Moreover, the European Food Safety Authority has recommended that energy drinks with caffeine content of more than $150 \mathrm{mg} / \mathrm{mL}$ should be labeled as "high caffeine content," in addition to the mention of the actual amount. However, the rule does not cover coffee, tea, and cocoa [18]. Owing to the observation of the above facts, caffeine estimation in beverages is essential to monitor its level in the safe limit and meet the regulatory standards. Several methods using a variety of analytical techniques have been used for the quantification of caffeine in beverages. These techniques include UV spectrophotometry [19], HPLC [15, 20], capillary electrophoresis [19], RP-UPLC [21], gas chromatography [22], GC-MS [1, 23, 24], continuous flow analyzer coupled with UV-Vis and evaporative light scattering detectors [17], solid phase-FT-Raman spectroscopy [25], and microemulsion electrokinetic chromatography [26].
However, most of the methods previously used for determination caffeine content in beverages usually involved complicated extraction procedures along with the usage of large amounts of organic solvents either in liquid extraction or in mobile phase. Moreover, some of the methods are expensive and time consuming. Consequently, a simple, fast, and ecofriendly method involving minimum organic solvent consumption for caffeine analysis in beverages is desirable. For several years, GC-MS has been considered as one of the most powerful instruments for separation and identification of analytes due to its high sensitivity and accuracy. Hence, in the present study, we have estimated the caffeine concentration in certain energy drinks available in Saudi Arabian market. We used a procedure which is inexpensive, fast, and utilizing least quantity of organic solvent, making the method ecofriendly. The method may be useful for beverage industries as well as regulatory authorities to monitor the caffeine content in beverages.

\section{Materials and Methods}

2.1. Samples and Reagents. Caffeine standard and carbamazepine (internal standard) were purchased from Sigma Aldrich, Germany. Nine commercially available caffeinated energy drink samples including Bugzy, Code Red, Shark, Red Bull, Bison Berry, Bison Super, W1 Energy drink, W1 Taurine, and Effect were purchased from local market of Jazan, Saudi Arabia, and were assigned codes as $\mathrm{D}_{1}, \mathrm{D}_{2}, \mathrm{D}_{3}$, $\mathrm{D}_{4}, \mathrm{D}_{5}, \mathrm{D}_{6}, \mathrm{D}_{7}, \mathrm{D}_{8}$, and $\mathrm{D}_{9}$, respectively. Methanol (99.9\%) and other chemicals were procured from Sigma Aldrich, Germany. All the reagents used in this study were of analytical grade. All the samples were stored in refrigerator until analysis. For each sample, only one manufacturer lot was analyzed in this experiment; hence, lot-to-lot variability was not evaluated. The sample analysis was carried out in triplicate $(n=3)$ and the average concentration has been reported.

2.2. Preparation of Solutions. Caffeine $(50 \mathrm{mg})$ was dissolved in $50 \mathrm{~mL}$ methanol to achieve standard stock solution of $1 \mathrm{mg} / \mathrm{mL}$ concentration in a volumetric flask. Similarly, $50 \mathrm{mg}$ of carbamazepine standard was transferred to a $50 \mathrm{~mL}$ volumetric flask and dissolved in methanol, and volume was adjusted with methanol to prepare a solution possessing $1 \mathrm{mg} / \mathrm{mL}$ concentration to be used as stock solution for internal standard. Measured volumes of caffeine standard stock solution were further diluted to obtained calibration standards over a range of $5-25 \mu \mathrm{g} / \mathrm{mL}$ ( 5 points levels). Stock solution of internal standard was then added to each calibration standard and enough methanol was added to complete the total volume (final concentration of internal standard was $10 \mu \mathrm{g} / \mathrm{mL}$ ). Quality control solutions of low, medium, and high concentration levels $(5,10$, and $20 \mu \mathrm{g} / \mathrm{mL}$ caffeine, respectively) were prepared from the above standard stocks. For preparation of sample solution, an aliquot of energy drink was directly taken from the product container and diluted 30 to 50 times in methanol $(10 \mu \mathrm{g} / \mathrm{mL}$, target concentration). For each product, the sample 
solutions were prepared from three different containers. An aliquot of $2 \mathrm{~mL}$ of each calibration standard and sample solution was transferred to GC vial and $2 \mu \mathrm{L}$ was injected into GC-MS system for analysis in triplicate. Methanol was used as blank.

2.3. Instrumentation. The GC-MS analysis was performed on gas chromatograph (Trace Ultra GC, Thermo Scientific, USA) attached with a mass spectrometric detector (ISQ, Thermo Fischer Scientific, USA) equipped with a single staged quadrupole and electron ionization source. The analytical separation was achieved on HP-5MS capillary GC column with $30 \mathrm{~m} \times 0.25 \mathrm{~mm}$ I.D. and $0.25 \mu \mathrm{m}$ film thickness. Helium with minimum purity of $99.9 \%$ was used as carrier gas, which has been maintained at a constant flow rate of $1.2 \mathrm{~mL} / \mathrm{min}$. The samples were injected in the system by using AI/AS 30000 autosampler equipped with $10 \mu \mathrm{L}$ syringe. The injector temperature was set at $280^{\circ} \mathrm{C}$ with mass transfer line temperature of $270^{\circ} \mathrm{C}$ and ion source temperature of $300^{\circ} \mathrm{C} .2 \mu \mathrm{L}$ of sample solutions was injected with splitless mode, and the syringe was washed with methanol three times before and after every injection and then rinsed with $8 \mu \mathrm{L}$ of sample solution before every injection. The GC column oven initial temperature was set at $70^{\circ} \mathrm{C}$ for 1 min hold time, ramped at a rate of $20^{\circ} \mathrm{C}$ up to $250^{\circ} \mathrm{C}$ and holding at this temperature for $5 \mathrm{~min}$. The maximum oven temperature was set at $300^{\circ} \mathrm{C}$ and equilibration time was $0.5 \mathrm{~min}$. The mass spectrometric analysis was carried out at electron impact mode $(70 \mathrm{eV}, \mathrm{EI})$, the full scan was performed over a range of $\mathrm{m} / z 100-550$, and $\mathrm{m} / z$ 194 and 236 were used for monitoring of caffeine and carbamazepine, respectively. Caffeine identification was done by direct comparison of retention time and mass spectrum of caffeine sample solutions with those in standard solution. The statistical analysis was performed by applying $T$-Test at $95 \%$ confidence interval level using MS Excel 2010. The test was performed to see whether the statistical difference between the mean analyzed concentration of caffeine in an energy drink sample and the label claim is significant $\left(P^{*}<0.05\right)$.

\section{Results and Discussion}

An analytical method for quantitative determination of caffeine in energy drinks was developed using gas chromatography and mass spectrometer as detector. Internal standard in an analysis is generally used to correct the systematic errors from the instrumental drift, random errors of repeatability of injection, and procedural errors. In this experiment, carbamazepine was selected as internal standard (IS) due to its retention similarity to caffeine. The retention time for caffeine and that for carbamazepine were 9.69 and $13.48 \mathrm{~min}$, respectively, with a total run time of 15 min. During the development process, different GC columns with different dimensions and stationary phases were tried and, finally, TR-5MS capillary GC column with $30 \mathrm{~m} \times 0.25 \mathrm{~mm}$ I.D. and $0.25 \mu \mathrm{m}$ film thickness was found to be optimum because sharp and symmetric peaks for both caffeine and IS were obtained. After several trials, the temperatures in the GC system were optimized and temperatures of $280^{\circ} \mathrm{C}$ and $300^{\circ} \mathrm{C}$ were set for injector port and column oven, respectively, to monitor the analytes. Similarly, the flow rate for helium was optimized by careful comparison of various flow rates and finally a flow rate of $1.2 \mathrm{~mL} / \mathrm{min}$ was adopted for optimum chromatograms. No solvent cutoff time was applied, as no interference from the sample matrix was observed in the initial run time. The mass spectrum of caffeine showed characteristic ion peak for caffeine at $\mathrm{m} / z 109.03$ and 194.23 and carbamazepine (IS) at $\mathrm{m} / z 193.26$ and 236.23. For quantification of caffeine and carbamazepine, ion peaks at $\mathrm{m} / z 194$ and 236, respectively, were monitored. Representative GC chromatograms of caffeine and carbamazepine (IS) obtained after optimization of all the chromatographic parameters are depicted in Figure 1.

\subsection{Method Validation}

3.1.1. Linearity, Limit of Detection, and Limit of Quantification. The linearity of the present method was evaluated by analyzing calibration standards over a range of $5-25 \mu \mathrm{g} / \mathrm{mL}$ of caffeine concentrations at five-point levels. The solutions were injected at an order from low to high concentration and the chromatograms were recorded. Calibration curve was constructed by plotting peak area ratios of caffeine and IS against caffeine concentration, and slope and intercept were calculated. A good linearity was achieved within the tested range with a correlation coefficient $\left(R^{2}\right)$ of 0.999 . The linear equation of calibration curve for caffeine was $y=0.2101 \mathrm{x}-0.6499$; where $y$ is the intercept and $x$ is the slope of the curve. LOD and LOQ values for caffeine were determined by using the standard deviation and slope of the above calibration curve. The LOD and LOQ values for this analysis were $0.53 \mu \mathrm{g} / \mathrm{mL}$ and $1.76 \mu \mathrm{g} / \mathrm{mL}$, respectively. The lower LOD and LOQ values have demonstrated the sensitivity of the method.

3.1.2. Precision and Accuracy. Intraday and interday precision were determined as \%RSD of caffeine peak areas and retention time of three replicate injections, and accuracy was evaluated by calculating the \%recovery of caffeine. For precision and accuracy experiment, the quality control solutions of low, medium, and high concentrations $(5,10$, and $20 \mu \mathrm{g} / \mathrm{mL}$, respectively) were analyzed. The intraday precision and accuracy was measured by carrying out analysis of quality control samples of each concentration at the same day, whereas the interday precision and accuracy was established by analyzing the quality control samples at three consecutive days (fresh samples prepared every day). The \% RSDs of analyte peak area for intra- and interday analysis were $<2 \%$ and the retention time of caffeine was found to be highly reproducible as the \%RSD of retention time in intraand interday analysis was also $<2 \%$. The calculated recoveries for intra- and interday analysis at three QC concentrations were in the range of $100 \pm 2 \%$. These results were 


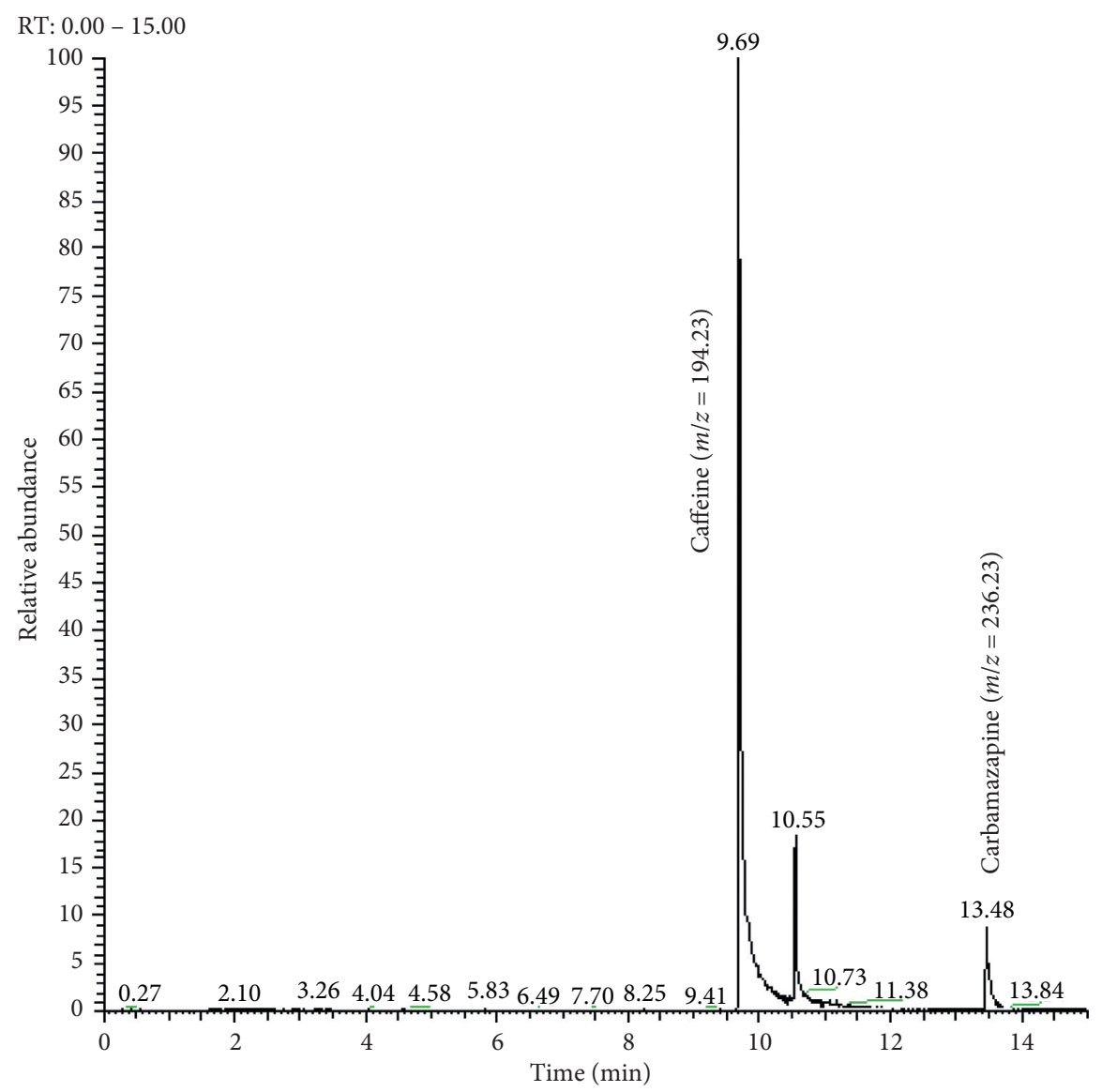

FigURE 1: Representative selective ion chromatogram of standard solution $(10 \mu \mathrm{g} / \mathrm{mL})$ showing caffeine and carbamazepine at $m / z 194$ and 236 , respectively.

within the acceptable limits of precision and accuracy and have conformed to the criteria of method validation according to the ICH guidelines. The observed values of precision and accuracy have proved that the present method was precise and accurate for caffeine analysis. The precision and accuracy results are summarized in Table 1.

3.1.3. Specificity. To examine the selectivity of the method, methanol was injected as blank and chromatograms were recorded. The bank chromatograms were compared with those obtained from calibration standards. No interference was observed at the retention time of caffeine and internal standard, suggesting that the method is specific. Furthermore, no carryover effect was observed throughout the analysis and all the peaks in standard as well as sample solutions were well resolved.

3.1.4. Solution Stability. The stability of caffeine in the analytical solution was examined by storing the caffeine working solution $(10 \mu \mathrm{g} / \mathrm{mL})$ at room temperature for $48 \mathrm{~h}$ for short-term stability, inside refrigerator $\left(\sim 4^{\circ} \mathrm{C}\right)$ for 7 days, and at $-20^{\circ} \mathrm{C}$ for 30 days to evaluate long-term stability. The working solutions were then analyzed by applying the optimized chromatographic condition. The assay analysis of stability solutions at the above conditions was recorded as
$99.04 \%, 99.18 \%$, and $99.26 \%$, respectively (Table 2). The results from all stability conditions demonstrated good stability of the analyte at each tested condition because no remarkable reduction $(<2 \%)$ in the caffeine concentration was found. Based on the results of stability experiments, it can be expected that there would be no stability related problem for caffeine in the analytical solutions during routine analysis.

3.1.5. Determination of Caffeine Content in Commercially Available Energy Drinks. The increasing popularity of energy drinks among young population and associated adverse effects on consumers' health have attracted the attention of scientific fraternity towards the scrutiny of these products. Although caffeine is the main active component, other ingredients such as guarana, taurine, vitamins, and ginseng are also present in energy beverages. Due to presence of these additional constituents, it has been suggested that the physiological effects of energy drinks may vary from other caffeine-containing beverages. Generally, the caffeine content is indicated on the dietary supplement label of the products; however, discrepancies were reported in the quantity of caffeine actually present in energy beverages with that of the label claim [27]. Therefore, in the current study, commercial energy drinks available in local Jazan market, 
TABle 1: Precision and accuracy results for intraday and interday analysis of caffeine by the proposed method.

\begin{tabular}{ccccc}
\hline & $\begin{array}{c}\text { Concentrations of solutions } \\
(\mu \mathrm{g} / \mathrm{mL})\end{array}$ & \% RSD of peak areas $(n=3)$ & \% RSD of retention times $(n=3)$ & $\%$ recovery $(n=3)$ \\
\hline \multirow{3}{*}{ Intraday analysis } & 5 (LQC) & 0.57 & 0.82 & 98.3 \\
& 10 (MQC) & 0.84 & 0.59 & 100.6 \\
\hline \multirow{3}{*}{ Interday analysis } & 20 (HQC) & 0.69 & 0.87 & 9.92 \\
& 5 (LQC) & 0.81 & 0.68 & 99.1 \\
& 10 (MQC) & 0.74 & 0.88 & 98.6 \\
\hline
\end{tabular}

*LQC, MQC, and HQC are low-, medium-, and high-quality control samples.

TABLE 2: Results of solution stability for caffeine.

\begin{tabular}{lcc}
\hline Analyte & Storage conditions & Mean recovery $\pm \% \mathrm{RSD}^{*}$ \\
\hline \multirow{3}{*}{ Caffeine } & $25 \pm 2^{\circ} \mathrm{C} ; 48 \mathrm{~h}$ & $99.04 \pm 0.0 .58$ \\
& $4-8^{\circ} \mathrm{C} ; 7$ days & $98.86 \pm 0.47$ \\
& $-20^{\circ} \mathrm{C} ; 30$ days & $99.26 \pm 0.0 .61$ \\
\hline
\end{tabular}

${ }^{*} n=3$. The caffeine working solution having a concentration of $10 \mu \mathrm{g} / \mathrm{mL}$ was used for solution stability experiment.

Saudi Arabia, were purchased. A total of nine samples of different brands were selected for investigation and for each brand three cans were collected. The sample solutions were prepared by aliquoting the content from each container separately by following the procedure described above and analyzed by the proposed GC-MS method. The suitability of the method was evaluated by performing the recovery study for caffeine through addition of known caffeine concentrations in the energy beverage sample solutions at $50 \%$, $100 \%$, and $150 \%$ levels of the target concentration $(10 \mu \mathrm{g} /$ $\mathrm{mL}$ ). After the analysis of recovery samples in triplicate, the $\%$ recovery of caffeine at each concentration level was found to be within $100 \pm 2 \%$ (Table 3 ). The good recovery results have indicated acceptable specificity of the analyte in the sample matrix. Furthermore, no alterations in the retention time and other system suitability parameters were observed during the analysis of the sample solutions (Figure 2).

According to the label claims, the amount of caffeine present in the energy drinks ranged between 20 and $32 \mathrm{mg} /$ $100 \mathrm{~mL}$ with an average amount of caffeine equal to $29.5 \mathrm{mg} /$ $100 \mathrm{~mL}$. Out of nine energy beverages, one mentioned $50 \mathrm{mg} /$ $250 \mathrm{~mL}(20 \mathrm{mg} / 100 \mathrm{~mL})$ of caffeine on its label $\left(\mathrm{D}_{3}\right.$, lowest $)$, while the amounts of caffeine claimed on the product label were 30 and $32 \mathrm{mg} / 100 \mathrm{~mL}$ for other four $\left(D_{1}, D_{2}, D_{4}\right.$, and $\left.D_{7}\right)$ and three $\left(D_{5}, D_{6}\right.$, and $\left.D_{9}\right)$ products, respectively. One energy beverage product $\left(\mathrm{D}_{8}\right)$ failed to specify the caffeine content on its product label. The caffeine content determined in this study was ranged between 20.82 and $33.72 \mathrm{mg} / 100 \mathrm{~mL}$ with an average value of $29.70 \mathrm{mg} / 100 \mathrm{~mL}$. The measured caffeine content in the tested products was in a range of 91.1-106.9\% of the label claims. All the products are being supplied in $250 \mathrm{~mL}$ pack size, except one $\left(D_{1}\right)$, which is being supplied in $185 \mathrm{~mL}$ pack size. The amount of caffeine estimated in this study along with label claim is presented in Table 4. The results reported in this study are average of three separate determinations. It is evident from Table 4 that, among the tested energy drink brands, $D_{5}$ contains highest caffeine concentration $(33.72 \mathrm{mg} / 100 \mathrm{~mL})$, while lowest caffeine content was detected in $\mathrm{D}_{3}(20.82 \mathrm{mg} / 100 \mathrm{~mL})$ against 30 and $20 \mathrm{mg} / 100 \mathrm{~mL}$ of caffeine content, respectively.

The caffeine concentrations determined in brands $\mathrm{D}_{6}$, $\mathrm{D}_{7}$, and $\mathrm{D}_{9}$ were less than their label claims $(93.8,94.9$, and $91.1 \%$, respectively) and products $D_{1}, D_{2}, D_{3}, D_{4}$, and $D_{5}$ were found to contain more caffeine than that mentioned on their product labels $(104.9,106.9,104.1,106.1$, and $105.4 \%$, respectively). The concentration of caffeine recorded in product $\mathrm{D}_{8}$, whose amount has not been specified in the dietary supplement label, was $29.16 \mathrm{mg} / 100 \mathrm{~mL}$. Overall, the amounts of caffeine were found in the following decreasing order: $D_{5}>D_{2}>D_{4}>D_{1}>D_{2}>D_{6}>D_{8}>D_{9}>D_{3}$. Two out of nine tested energy beverage brands were found to contain caffeine, within $100 \pm 5 \%$ (approximately $\pm 5 \%$ deviation) of what was indicated on the product label, whereas within $100 \pm 10 \%$ (within $\pm 10 \%$ deviation) of the labeled caffeine was detected in six other products mentioning the amount of caffeine on their dietary supplement labels. Although the variations from the labeled caffeine concentrations in energy beverages were also reported previously by other studies, the deviations observed in our investigations were found to be less. Furthermore, as per the label claim, the amount of caffeine was significantly lower $(20-32 \mathrm{mg} / 100 \mathrm{~mL}$ or 50 $80 \mathrm{mg} / \mathrm{pack})$ in the product screened in the present investigation. Attipoe et al. (2016) determined the caffeine content in certain energy drinks and reported approximately $\pm 15 \%$ deviation from the label claims [28]. In a similar study, Cohen et al. (2013) found that the quantity of caffeine in $45 \%$ of the tested dietary supplements varied by $\pm 10 \%$ of the labeled amount, whereas $25 \%$ of the products were found to have wider variations [27]. Another study reported that the determined amounts of caffeine in the majority of products were within $\pm 20 \%$ of the amount declared on the dietary supplement label [29]. According to the regulation of the US Food and Drug Administration, the dietary ingredients specifically added in a product must contain $100 \%$ of the weight/volume declared on the product label (with deviation due to analytical errors) [30]. This means that the energy beverages tested in this study slightly deviated from criteria set by the USFDA for amount of caffeine mentioned on the product label and actually present. Furthermore, the majority of the products tested in previous studies were also unable to meet the FDA requirement with respect to the caffeine concentration.

Although it can be recommended that occasional intake of caffeinated energy drinks may be acceptable for adult population, it would be most appropriate to select one that 
TABLE 3: Recovery analysis results of caffeine in commercial energy beverage samples.

\begin{tabular}{lccc}
\hline $\begin{array}{l}\text { Concentration of recovery } \\
\text { samples }(\mu \mathrm{g} / \mathrm{mL})\end{array}$ & $\begin{array}{c}\text { Percent of target concentration } \\
(\mu \mathrm{g} / \mathrm{mL})(\%)^{*}\end{array}$ & $\begin{array}{c}\text { Average amount of caffeine } \\
\text { recovered }(\mu \mathrm{g} / \mathrm{mL})^{* *}\end{array}$ & $\begin{array}{c}\text { Average percent } \\
\text { recovery } \pm \% \mathrm{RSD}^{* *}\end{array}$ \\
\hline 5 & 50 & 5.09 & $101.8 \pm 0.20$ \\
10 & 100 & 9.92 & $99.2 \pm 1.23$ \\
15 & 150 & 15.16 & $101.1 \pm 0.66$ \\
\hline
\end{tabular}

${ }^{*}$ Target concentration is $10(\mu \mathrm{g} / \mathrm{mL}) .{ }^{* *}$ Average of three determinations.

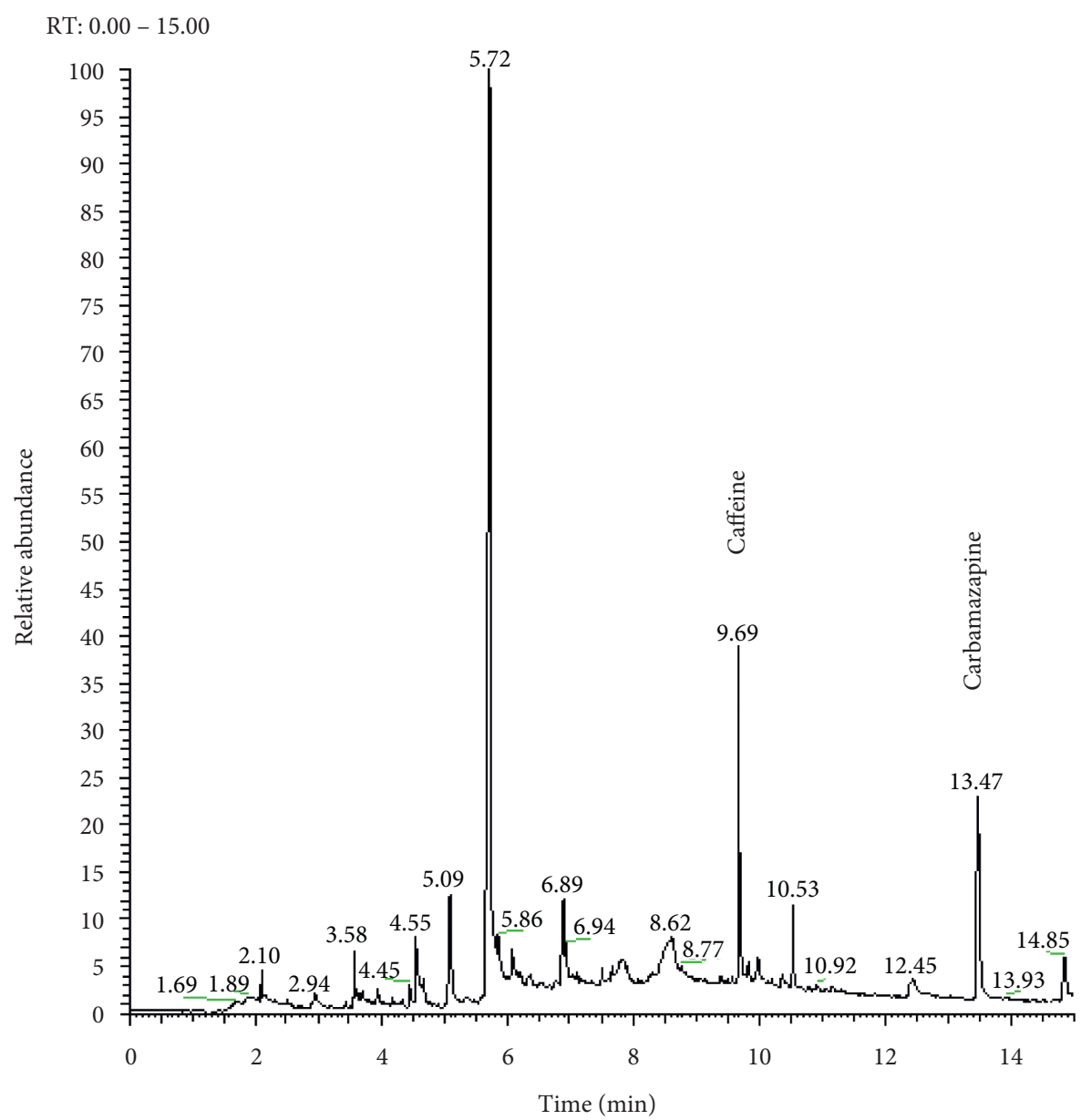

(a)

FIgURE 2: Continued. 


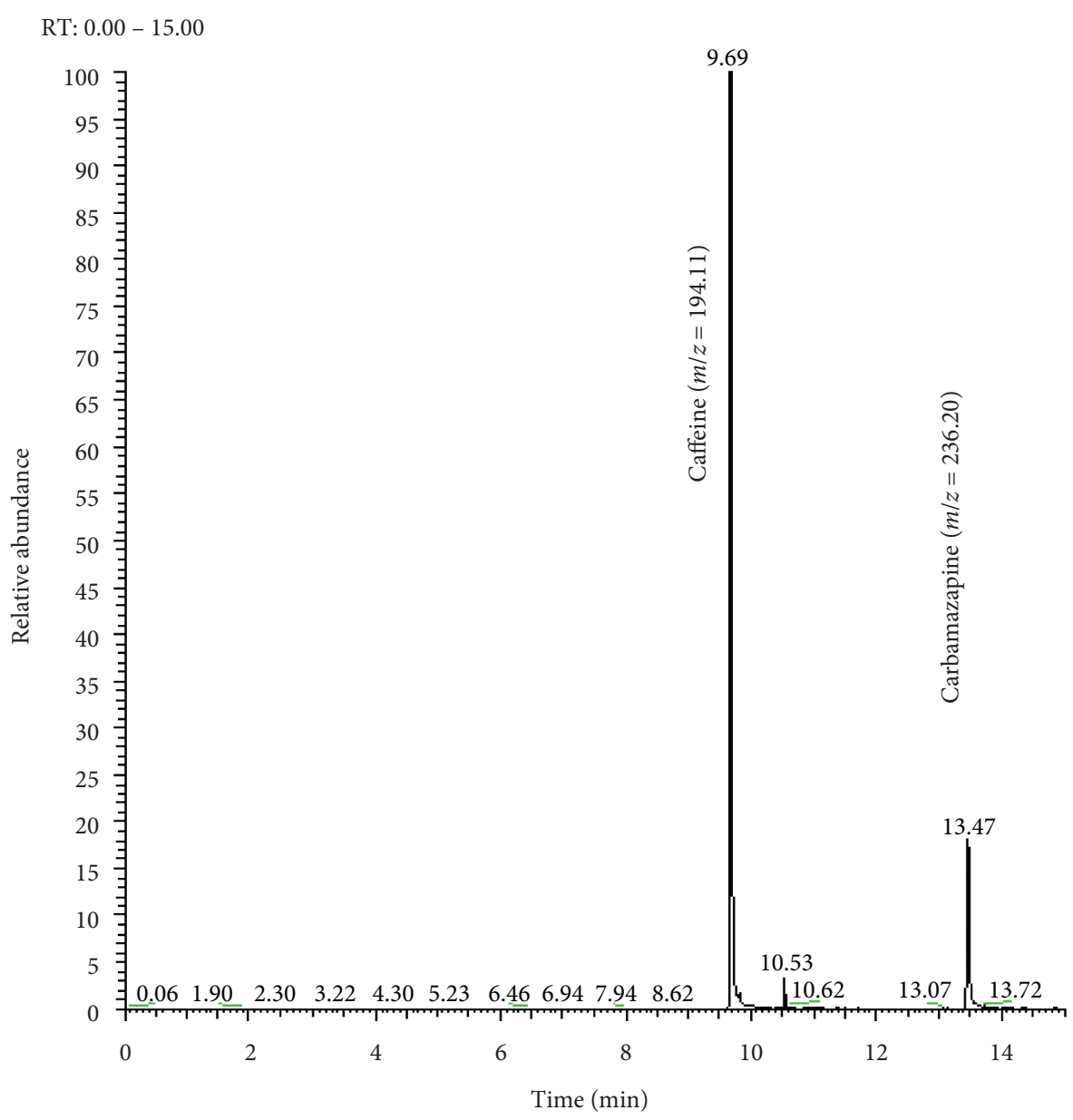

(b)

FIGURE 2: Representative chromatogram showing caffeine and carbamazepine (IS) peaks in the energy drink sample analyzed by the proposed GC-MS method: (a) total chromatogram and (b) selected ion chromatogram (concentration $\approx 10 \mu \mathrm{g} / \mathrm{mL}$ ).

TABLE 4: Concentration of caffeine in energy beverages collected from Saudi Arabian market, determined by the current GC-MS method.

\begin{tabular}{lcccc}
\hline Energy drink sample & $\begin{array}{c}\text { Caffeine content as label claim } \\
(\mathrm{mg} / 100 \mathrm{~mL})\end{array}$ & Pack size $(\mathrm{mL})$ & $\begin{array}{c}\text { Caffeine content detected } \\
(\mathrm{mg} / 100 \mathrm{~mL}) \pm \mathrm{SD}\end{array}$ & Percent of the label claim \\
\hline $\mathrm{D}_{1}$ & 30 & 185 & $31.47 \pm 0.69$ & 104.9 \\
$\mathrm{D}_{2}$ & 30 & 250 & $32.08 \pm 0.97$ & 106.9 \\
$\mathrm{D}_{3}$ & 20 & 250 & $20.82 \pm 1.21$ & 104.1 \\
$\mathrm{D}_{4}$ & 30 & 250 & $31.82 \pm 0.82$ & 106.1 \\
$\mathrm{D}_{5}$ & 32 & 250 & $33.72 \pm 0.82$ & 105.4 \\
$\mathrm{D}_{6}$ & 32 & 250 & $30.03 \pm 0.83$ & 93.8 \\
$\mathrm{D}_{7}$ & 30 & 250 & $28.48 \pm 1.67$ & - \\
$\mathrm{D}_{8}$ & Not mentioned & 250 & $29.75 \pm 1.21$ & 94.9 \\
$\mathrm{D}_{9}$ & 32 & 250 & $29.16 \pm 1.11^{*}$ & 91.1 \\
\hline
\end{tabular}

SD: standard deviation $(n=3)$. The statistical difference between the detected mean caffeine contents and label claims of respective energy drink samples was analyzed by the $T$-test at $95 \%$ confidence level $\left({ }^{*} P<0.05\right)$.

mentions the quantity of caffeine on its label, because the product that does not specify the amount may contain higher caffeine content (up to $300 \mathrm{mg} / 100 \mathrm{~mL}$ ). Moreover, the discrepancies were reported in the quantity of caffeine actually present in energy beverages with that claimed on the dietary supplement label of the products [27]. Approximately, $400 \mathrm{mg}$ per day caffeine consumption is considered to be safe upper limit for healthy adults, whereas this limit is $200 \mathrm{mg}$ per day for pregnant women and 45-85 mg per day for children depending on their body weights $[28,31]$. However, the intake of caffeine by children and adolescents is being discouraged by the American Academy of Pediatrics. According to the USFDA, rapid consumption of about $1200 \mathrm{mg}$ of caffeine or 0.15 
tablespoons of pure caffeine may produce major toxic effects such as seizures [32]. The caffeine concentrations recorded in the tested energy drinks were within the prescribed maximum limit (less than $50 \mathrm{mg} / 100 \mathrm{~mL}$ ), which is unlikely to pose any adverse effect to the consumer population. Moreover, the amounts of caffeine observed in the current investigation were lower than those recorded in previous studies and so the label claims. The caffeine intake through consumption of energy drink is generally an addition to the amount ingested from other sources such as coffee and tea. Moreover, some individuals consume multiple servings of energy drinks per day. As a result, cumulative amount of caffeine intake may exceed the upper limit and could lead to overdose or intoxication.

According to information received from the emergency ward of a hospital in Saudi Arabia, a young adult was admitted with symptoms of nervousness, anxiety, agitation, insomnia, and palpitation. The exact reason for the emergency condition was not known; however, the patient had taken two tablets containing $65 \mathrm{mg}$ caffeine along with $500 \mathrm{mg}$ paracetamol (Panadol Extra effervescent tablet) after dissolving in energy drink. This was in addition to intake of tea/coffee, which may have resulted in overdose of caffeine or other ingredients leading to symptoms encountered by the patient. Hence, the consumers should be aware that the energy drinks contain caffeine as one of the ingredients and that the actual amount of caffeine in energy drinks may vary from that indicated in the product label. Also, the health care practitioner should be aware of the extent of usage of energy drinks in the society and their associated adverse impact on human health, so that they can appropriately counsel the patients.

\section{Conclusion}

In this study, caffeine content in nine most popular energy drinks marketed in Saudi Arabia was determined by GC-MS analysis. The analytical method was developed and validated and found to be linear, precise, accurate, and specific. The analysis was simple, as the samples were directly injected after multifold dilutions in methanol. The caffeine concentrations in the tested energy beverages were $20.82-33.72 \mathrm{mg} / 100 \mathrm{~mL}$. The highest caffeine content was recorded in product $D_{5}$, whereas the lowest concentration was found to be in product $\mathrm{D}_{3}$. The caffeine content in five out of nine products was recorded higher than the respective labeled amounts (104.1-106.9\%), whereas lower caffeine concentrations were observed in three other tested energy beverages (91.1-94.9\% of the label claim). Overall, the amounts of caffeine present in the tested energy drinks were within the safe upper limit of daily caffeine consumption. The caffeine concentrations in the tested beverages were within $100 \pm 10 \%$ of the label claim (less than $10 \%$ deviation from the label claim). On the basis of the results obtained in this study, the manufacturers of these beverages are suggested to be more accurate about specifying the contents of the ingredients added. To avoid adverse health effects, the consumers should consume caffeine below upper safe limit prescribed by the regulatory agencies such as FDA and should avoid the products failing to mention the amount on the dietary supplement label.

\section{Data Availability}

The data used to support the findings of this study are available from the corresponding author upon request.

\section{Conflicts of Interest}

The authors declare that there are no conflicts of interest related to this manuscript.

\section{Acknowledgments}

This work was financially supported by the Deanship of Scientific Research, Jazan University, Jazan, Saudi Arabia (Grant number: SARC/002).

\section{References}

[1] J. Ayala, K. Simons, and S. Kerrigan, "Quantitative determination of caffeine and alcohol in energy drinks and the potential to produce positive transdermal alcohol concentrations in human subjects," Journal of Analytical Toxicology, vol. 33, no. 1, pp. 27-33, 2009.

[2] J. L. Harris and C. R. Munsell, "Energy drinks and adolescents: what's the harm?" Nutrition Reviews, vol. 73, no. 4, pp. 247-257, 2015.

[3] M. A. Heckman, K. Sherry, and E. G. De Mejia, "Energy drinks: an assessment of their market size, consumer demographics, ingredient profile, functionality, and regulations in the United States," Comprehensive Reviews in Food Science and Food Safety, vol. 9, no. 3, pp. 303-317, 2010.

[4] J. L. Reid, C. McCrory, C. M. White et al., "Consumption of caffeinated energy drinks among youth and young adults in Canada," Preventive Medicine Reports, vol. 5, pp. 65-70, 2017.

[5] K. A. Clauson, K. M. Shields, C. E. McQueen, and N. Persad, "Safety issues associated with commercially available energy drinks," Journal of the American Pharmacists Association, vol. 48, no. 3, pp. e55-e67, 2008.

[6] F. Ali, H. Rehman, Z. Babayan, D. Stapleton, and D.-D. Joshi, "Energy drinks and their adverse health effects: a systematic review of the current evidence," Postgraduate Medicine, vol. 127, no. 3, pp. 308-322, 2015.

[7] United State Food and Drug Administration (USFDA), "Energy "drinks" and supplements: investigations of adverse event reports," 2012, https://www.fda.gov/Food/News Events/ ucm328536.htm.

[8] N. Gunja and J. A. Brown, "Energy drinks: health risks and toxicity," Medical Journal of Australia, vol. 196, no. 1, pp. 46-49, 2012.

[9] S. Azagba, D. Langille, and M. Asbridge, "An emerging adolescent health risk: caffeinated energy drink consumption patterns among high school students," Preventive Medicine, vol. 62, pp. 54-59, 2014.

[10] N. Larson, M. N. Laska, M. Story, and D. Neumark-Sztainer, "Sports and energy drink consumption are linked to healthrisk behaviours among young adults," Public Health Nutrition, vol. 18, no. 15, pp. 2794-2803, 2015.

[11] Y. M. Terry-McElrath, P. M. O’Malley, and L. D. Johnston, "Energy drinks, soft drinks, and substance use among United 
States secondary school students," Journal of Addiction Medicine, vol. 8, no. 1, pp. 6-13, 2014.

[12] S. J. P. Iyadurai and S. S. Chung, "New-onset seizures in adults: possible association with consumption of popular energy drinks," Epilepsy \& Behavior, vol. 10, no. 3, pp. 504-508, 2007.

[13] J. Chelben, A. Piccone-Sapir, I. Ianco, N. Shoenfeld, M. Kotler, and R. D. Strous, "Effects of amino acid energy drinks leading to hospitalization in individuals with mental illness," General Hospital Psychiatry, vol. 30, no. 2, pp. 187-189, 2008.

[14] G. Richards and A. Smith, "Caffeine consumption and selfassessed stress, anxiety, and depression in secondary school children," Journal of Psychopharmacology, vol. 29, no. 12, pp. 1236-1247, 2015.

[15] R. Hartyel, I. J. Smith, and J. R. Cookman, "Improved HPLC method for the simultaneous determination of caffeine and its $\mathrm{N}$-demethylated metabolites in plasma using solid-phase extraction," Journal of Chromatography A, vol. 324, pp. 105-117, 1985.

[16] P. W. Curatolo and D. Robertson, "The health consequences of caffeine," Annals of Internal Medicine, vol. 98, no. 5_Part_1, pp. 641-653, 1983.

[17] R. Lucena, S. Cárdenas, M. Gallego, and M. Valcárcel, "Continuous flow autoanalyzer for the sequential determination of total sugars, colorant and caffeine contents in soft drinks," Analytica Chimica Acta, vol. 530, no. 2, pp. 283-289, 2005.

[18] European Food Safety Authority, "EFSA panel on dietetic products, nutrition and allergies (NDA), "scientific opinion on the safety of caffeine," EFSA Journal, vol. 13, no. 5, p. 4102, 2015.

[19] V. L. McDevitt, A. Rodriguez, and K. R. Williams, “Analysis of Soft Drinks: UV spectrophotometry, liquid chromatography, and capillary electrophoresis," Journal of Chemical Education, vol. 75 , no. 5 , p. $625,1998$.

[20] A. Gliszczynska-Swiglo and I. Rybicka, "Simultaneous determination of caffeine and water-soluble vitamins in energy drinks by HPLC with photodiode array and fluorescence detection," Food Analytical Methods, vol. 8, pp. 139-146, 2015.

[21] F. Turak, R. Güzel, and E. Dinç, "Simultaneous determination of ascorbic acid and caffeine in commercial soft drinks using reversed-phase ultraperformance liquid chromatography," Journal of Food and Drug Analysis, vol. 25, no. 2, pp. 285-292, 2017.

[22] H. J. Lin, M. L. Wang, C. W. Chen, B. S. Hwang, M. H. Lee, and Y. M. Choong, "A gas chromatographic method for determination of nicotinamide, paraben esters and caffeine in commercial health drinks, tonic drinks and cold formulas," Journal of Food and Drug Analysis, vol. 8, pp. 180-186, 2000.

[23] J. G. Lisko, E. Grace, J. Lee et al., "Caffeine concentrations in coffee, tea, chocolate, and energy drink flavored e-liquids," Nicotine \& Tobacco Research, vol. 19, no. 4, pp. 484-492, 2017.

[24] J. Zou and N. Li, "Simple and environmental friendly procedure for the gas chromatographic-mass spectrometric determination of caffeine in beverages," Journal of Chromatography A, vol. 1136, no. 1, pp. 106-110, 2006.

[25] S. Armenta, S. Garrigues, and M. de la Guardia, "Solid-phase FT-Raman determination of caffeine in energy drinks," Analytica Chimica Acta, vol. 547, no. 2, pp. 197-203, 2005.

[26] E. Liotta, R. Gottardo, C. Seri et al., "Rapid analysis of caffeine in smart drugs and energy drinks by microemulsion electrokinetic chromatography (MEEKC)," Forensic Science International, vol. 220, no. 1-3, pp. 279-283, 2012.

[27] P. A. Cohen, S. Attipoe, J. Travis, M. Stevens, and P. Deuster, "Caffeine content of dietary supplements consumed on military bases," JAMA Internal Medicine, vol. 173, no. 7, pp. 592-594, 2013.

[28] S. Attipoe, J. Leggit, and P. A. Deuster, "Caffeine content in popular energy drinks and energy shots," Military Medicine, vol. 181, no. 9, pp. 1016-1020, 2016.

[29] K. W. Andrews, A. Schweitzer, C. Zhao et al., "The caffeine contents of dietary supplements commonly purchased in the US: analysis of 53 products with caffeine-containing ingredients," Analytical and Bioanalytical Chemistry, vol. 389, no. 1, pp. 231-239, 2007.

[30] United State Food and Drug Administration, "Dietary supplement labeling guide: chapter IV. Nutrition labeling," 2005, https://www.fda.gov/Food/GuidanceRegulation/GuidanceDo cumentsRegulatoryInformation/Dietary Supplements/ucm07 0597.htmAvailable at.

[31] K. A. Sepkowitz, "Energy drinks and caffeine-related adverse effects," JAMA, vol. 309, no. 3, pp. 243-244, 2013.

[32] United State Food and Drug Administration, "Consumer updates. foods and beverages spilling the beans: how much caffeine is too much?," 2018, http://www.fda.gov/consumers/ consumer-updates/spilling-beans-how-much-caffeine-toomuch. 